\title{
Enhanced Distributed Dynamic Skyline Query for Wireless Sensor Networks
}

\author{
Khandakar Ahmed ${ }^{1,2,3, *}$, Nazmus S. Nafi ${ }^{4,+}$ and Mark A. Gregory ${ }^{4,+}$ \\ 1 School of Electrical and Computer Engineering, RMIT University, Melbourne, VIC 3000, Australia \\ 2 Melbourne Institute of Technology, Melbourne VIC 3000, Australia \\ 3 Department of Computer Science \& Engineering, Shahjalal University of Science \& Technology, \\ Sylhet-3114, Bangladesh \\ 4 School of Electrical and Computer Engineering, RMIT University, Melbourne, VIC 3000, Australia; \\ nazmusshaker.nafi@rmit.edu.au (N.S.N.); mark.gregory@rmit.edu.au (M.A.G.) \\ * Correspondence: khandakar.ahmed@rmit.edu.au or kahmed@academic.mit.edu.au or tanvir-cse@sust.edu; \\ Tel.: +61-3-9925-1180 (ext. 51180) \\ + These authors contributed equally to this work.
}

Academic Editor: Stefan Fischer

Received: 16 November 2015; Accepted: 28 January 2016; Published: 3 February 2016

\begin{abstract}
Dynamic skyline query is one of the most popular and significant variants of skyline query in the field of multi-criteria decision-making. However, designing a distributed dynamic skyline query possesses greater challenge, especially for the distributed data centric storage within wireless sensor networks (WSNs). In this paper, a novel Enhanced Distributed Dynamic Skyline (EDDS) approach is proposed and implemented in Disk Based Data Centric Storage (DBDCS) architecture. DBDCS is an adaptation of magnetic disk storage platter consisting tracks and sectors. In DBDCS, the disc track and sector analogy is used to map data locations. A distance based indexing method is used for storing and querying multi-dimensional similar data. EDDS applies a threshold based hierarchical approach, which uses temporal correlation among sectors and sector segments to calculate a dynamic skyline. The efficiency and effectiveness of EDDS has been evaluated in terms of latency, energy consumption and accuracy through a simulation model developed in Castalia.
\end{abstract}

Keywords: wireless sensor networks; distributed data centric storage; skyline query; sector based distance routing; uniform distribution; lower bound distribution; and upper bound distribution

\section{Introduction}

WSNs have successfully been validated as a very economic and effective platform for monitoring diverse physical environments from remote locations. Various types of query techniques have been developed over WSNs including min-max [1], top-k [2,3] and skyline [4]. Skyline and its variants such as traditional skyline [5] and dynamic skyline [6-8] have been applied in many multiple criteria decision making applications. Traditional skyline (TS) retrieves all of the points, which are not dominated by others, from a set of points [9]. Given a dataset $X$, a point $x_{1}$ dominates $x_{2}$, if $x_{1}$ is not worse than $x_{2}$ for each dimension $i \in l$ (i.e., $x_{1}[i] \leqslant x_{2}[i]$ ), and $x_{1}$ is better than $x_{2}$ for at least one dimension $m \in l\left(x_{1}[m]<x_{2}[m]\right)$. Figure 1a shows an example, where each point is drawn taking two dimensions $i$ and $j$ as coordinators; points $x_{1}, x_{3}, x_{6}$ and $x_{10}$ are in the skyline set considering that a point with the least value for each dimension is desirable.

Dynamic skyline (DS) retrieves a set of points that are not dynamically dominated by others with respect to a data point $q$ denoted by DS $(q, X))$ [10]. A point $x_{1}$ dynamically dominates $x_{2}$ with respect to $q$ if for each dimension in $i \in l,\left|x_{1}[i]-q[i]\right| \leqslant\left|x_{2}[i]-q[i]\right|$ and for at least one dimension in $m \in l$, 
$\left|x_{1}[m]-q[m]\right|<\left|x_{2}[m]-q[m]\right|$. In Figure $1 \mathrm{~b}$, points $x_{1}, x_{2}$ and $x_{4}$ are the dynamic skyline points of $q$. Each point $x_{i}=\left(x_{i}[j], x_{i}[i]\right)$ is transformed to $x_{i}{ }^{\prime}=\left(x_{i}[j]-q[j], x_{i}[i]-q[i]\right)$.

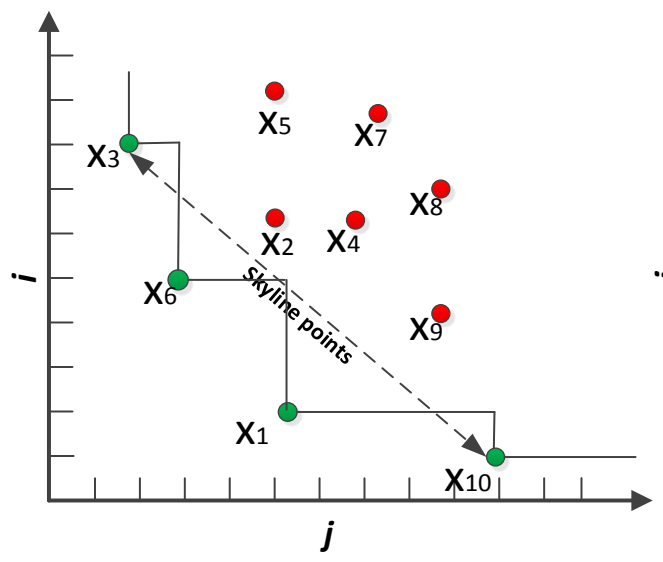

(a)

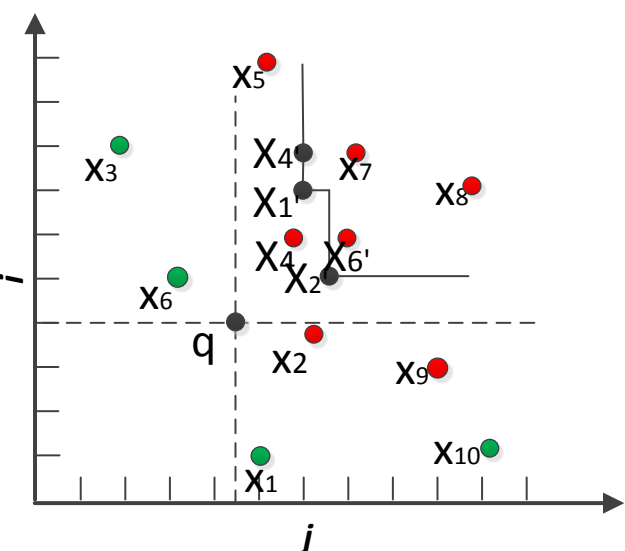

(b)

Figure 1. (a) TS and (b) DS ( $q, X)$.

Skyline query is very useful in multi-preference analysis and decision making for environmental monitoring applications. For example, in a WSN deployed in a forest with each sensor node sensing temperature and wind speed, it is possible to build a fast and energy efficient forest fire detection method [11] by concentrating on places with high temperature or fast wind speed, or both. To illustrate the idea of dominance relations in this scenario, consider the readings in the two-dimensional attribute space shown in Figure 2 depicting a typical example of ranking objects by more than one criterion. Figure 2a lists nine records received from nine corresponding sensor nodes deployed in different places and their values. Figure $2 \mathrm{~b}$ depicts the representation in a $2 \mathrm{D}$ space. Places $\mathrm{P}_{1}, \mathrm{P}_{2}, \mathrm{P}_{3}, \mathrm{P}_{5}$, and $\mathrm{P}_{6}$ are all dominated by other points so the skyline query returns the points that are not dominated by any other points. Consider the point $P_{5}$ that is dominated by $P_{7}$, as it has a higher wind speed than $P_{5}$ though both have the same temperature. The skyline query only retrieves the dominant places in terms of higher temperature and wind speed. The skyline query will not exhibit any place with lower temperature and wind speed once it is dominated by a location with higher values. Therefore, the result set of the skyline query consists of $\left\{\mathrm{P}_{4}, \mathrm{P}_{7}, \mathrm{P}_{8}, \mathrm{P}_{9}\right\}$, which are indicators of dangerous places that need special attention.

\begin{tabular}{|c|c|c|}
\hline Points & Temperature & Wind speed \\
\hline P1 & 1 & 9 \\
\hline P2 & 2 & 6 \\
\hline P3 & 5 & 3 \\
\hline P4 & 9.5 & 1.5 \\
\hline P5 & 9.0 & 4.5 \\
\hline P6 & 6 & 7 \\
\hline P7 & 9 & 8 \\
\hline P8 & 7 & 9 \\
\hline P9 & 5 & 10 \\
\hline
\end{tabular}

(a)

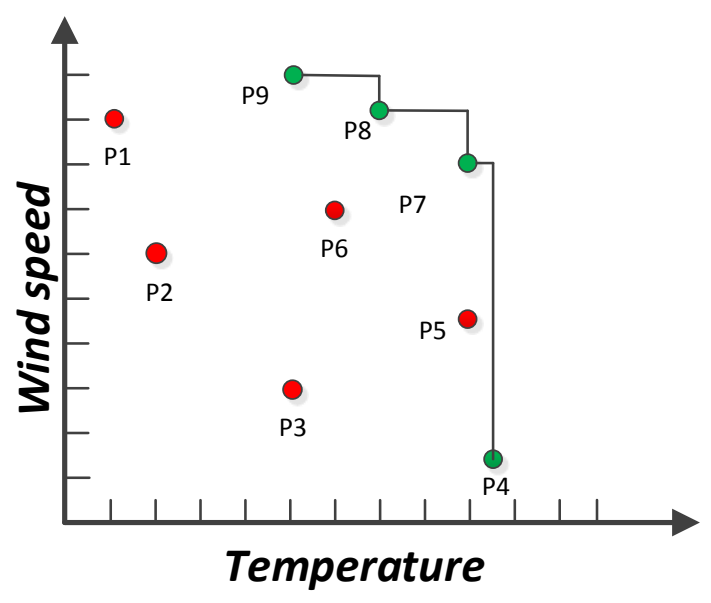

(b)

Figure 2. (a) Skyline of sensor readings, (b) Representation of sensor readings in 2D space [11]. 
Another example that demonstrates the usefulness of DS can be found when monitoring the air pollution in a region of interest. A high concentration of $\mathrm{CO}$ or $\mathrm{SO}_{2}$, or both, can be a strong indication that the location is highly polluted. An environmental scientist can issue a dynamic skyline query for $\mathrm{CO}$ and $\mathrm{SO}_{2}$ levels to monitor the air pollution surrounding a particular region of interest.

DS query processing over moving objects is also very useful for numerous applications, such as location-aware computing, object tracking and monitoring, virtual environments, uncertain data stream, computer games, visualization, etc.

Although the skyline query approach has potential for use in WSN applications, it faces challenges because of high processing complexity and cost related with updating the results. The main contributors to the high cost are the data access cost from storage locations and the processing cost while executing the user specified query for a dominance check. It is important to note that search efficiency and update criteria are two key requirements of skyline query processing and skyline result maintenance.

Data-Centric Storage (DCS) [12,13], an alternate to External Storage (ES) and Local Storage (LS), is considered to be a promising and efficient storage and search mechanism. There has been a growing interest in understanding and optimizing WSN DCS schemes in recent years, where a various number of query mechanisms such as point query, range query, similarity searching, top-k query and skyline query can be applied in a consolidated framework. As part of developing such consolidated framework, this paper proposes a threshold based hierarchical approach, which uses temporal correlation among the sectors and sector segments to calculate DS in a region of interest. To our knowledge, this is the first practical demonstration of DS in DCS of WSN in current state-of-the-art.

In this paper, first a novel tree building algorithm is incorporated so that each head node of a cluster could construct a tree with itself as a root. This allows exertion of three core operations such as Tree Propagation, Regular Update and Triggered Query from any head node with a particular range. The performance of the proposed model is analyzed by framing and implementing it in a distributed information delivery service running one or more applications in a WSN. In this service, a set of producer and consumer nodes exchange information by relaying packets through neighboring clusters for each application. This phenomenon is facilitated by a DCS [14] architecture, also referred to as DBDCS (Section 3.1). DBDCS is an adaptation of the magnetic disk storage platter consisting of tracks and sectors $[15,16]$. In DBDCS, each application uses a disc track and sector analogy to map data locations and uses distance based indexing method for storing and querying multi-dimensional similar data. The member nodes in each cluster report the sensed event to their associated head node, which aggregates the received events at the end of each epoch. The aggregated event is hashed to produce a hash key, which is mapped from a one dimensional domain into a metric space [17] (Section 3.3).

The remainder of this paper is structured as follows: Section 2 provides an overview of the related work in the literature. Network architecture, data processing and mapping, insertion and skyline querying are illustrated in Section 3. This is followed by the simulation results and performance evaluation of EDDS presented in Section 4. The paper is concluded in Section 5.

\section{Related Work}

According to the current state-of-the-art, skyline operator in database community was first introduced by Borzsonyi et al. [5]. In their work, they proposed the solution based on block nested loop (BNL) and divide-and-conquer (D \& C). Inspired by BNL, Chomicki et al. [18] and Godfrey et al. [19] proposed two variants of BNL named sort-filter-skylines (SFS) and linear elimination sort for skyline (LESS), respectively. Later, two progressive processing algorithms such as Bitmap and Index were proposed by Tan et al. [20]. Nearest neighbor (NN) method and its variant NN with branch-and-bound (BB) were later presented by Kossman et al. [21] and Papadias et al. [22], respectively. However, these approaches are mainly suitable for centralized environments with high computational and energy resources and thus inappropriate for WSN environments. 
A filter-based distributed algorithm for skyline evaluation and maintenance in WSN is proposed by Liang et al. [23]. Each sensor node uses a greedy algorithm to compute a local skyline certificate, which is a subset of the local skyline and forwards it to its parent. The parent or non-leaf node calculates the certificate based on a set of local skyline points plus the certificate received from child nodes. Following this process, the root calculates the set of skyline points, which is used as a Global Skyline Filter (GSF) or global certificate. The root broadcasts the global certificate followed by a simple merge-based algorithm to filter out the points from transmissions that cannot utilize the certificate. An energy efficient Sliding Window Skyline Monitoring Algorithm (SWSMA) is proposed in [24] to continuously maintain sliding window skylines over WSN. It reduces the amount of data transferred and reduces energy consumption, as a consequence, by devising two filter based algorithms: (1) Single point filter based algorithm referred to as a Tuple Filter (TF); and (2) Grid Filter (GF) based algorithm. Chen et al. [25] proposed two evaluation algorithms for finding the skyline points on a dataset progressively. The dataset is first partitioned into disjoint subsets. Then skyline points are returned through the examination of each subset progressively using discovered skyline points to filter out the unlikely skyline points from transmission. However, the features of DCS have not been considered in these approaches and thus direct implication of them in DCS is not suitable.

Song et al. [26] propose a skyline query processing algorithm exploiting the key features of DCS. The algorithm processes a query in three stages on the basis of four assumptions: (1) choice of DCS framework is limited to KDDCS [27], GDCS [28] and DIM [29]; (2) all nodes are identified by unique ID; (3) each node knows the geographic location of its own and its neighbor nodes; and (4) each node knows the range of data stored in its neighbor nodes. In the first stage, the base station locates the Start Node (SN) where the query is to commence. In the second stage, SN creates a Skyline Query Message (SQM) and propagates the SQM to neighbor nodes to search for Candidate Nodes (CNs) where candidate data for the query are stored. This SQM transmission process is repeated until the complete candidate results are found. In the third and final stage BS generates a final query result from its gathered candidate dataset. The aforementioned assumptions made in this work are particularly expensive in a distributed environment like WSN especially DCS framework.

Su et al. [30] proposed an algorithm, known as Skyline Sensor Algorithm (SkySensor), in a customized DCS method in order to collect and store all sensor readings and retrieve skyline results efficiently from the network. The major disadvantages of SkySensor are: (1) SkySensor needs increased effort from an application designer who has to write the center location of each cluster in such a way that no two clusters overlap; (2) number of clusters in a sensor network depends on the number of attributes of a tuple, therefore, an active sensor and actuator network, with higher rate of data generation and a lower number of attributes leads to high concentration of data in a small portion of the network which creates congestion and a hot spot around the cluster and limits the ultimate goal or advantage of DCS; and (3) in the case of resilience to node failure, an inefficient and old technique referred to as local replication is used, which is expected to incur high storage cost and increased data loss during a node group failure.

Based on the above discussion, it is obvious that none of the work from the current literature has implemented DS in WSN, especially in DCS. It consumes great effort in pre-processing, such as gathering the local skylines of all sensors, mapping all detected data of the entire network (both inflow and outflow). The overall process is overwhelmed with higher overheads and complexity if the researcher or analysts are interested in a particular region. In this paper, a threshold based hierarchical DS approach is proposed and implemented using the temporal correlation among the sectors and sector segments. This allows reducing the transmission cost in a greater aspect and facilitates finding skyline points from both entire network and a particular region of interest.

\section{Basic System Design}

In this section, at first, we briefly discuss the DBDCS network architecture that has been used for testing our proposed EDDS approach. Definition of skyline is restated in Section 3.2 in terms of 
metric based searching. This is followed by the discussion of data processing, mapping and insertion technique in Sections 3.3 and 3.4. Finally, we have presented the EDDS approach in Section 3.5.

\subsection{Network Architecture}

The surface/platter of a magnetic disk storage device consisting of tracks and sectors provides an interesting approach that may be applied to a large scale WSN. This assumption led to the Disk Based Data Centric Storage (DBDCS) architecture, as shown in Figure 3a, dividing the rectangular field into a matrix of storage cells (referred to as a sector) where row and column represent track $T_{i}$ and sector $S_{j}$, respectively. The physical deployment is mapped to a $m x n$ matrix, where $m$ is the number of tracks and $n$ is the number sectors for each track. Hence, the nodes in the network are divided into $S(m x n)$ sectors, each comprising a Sector Head (SH) and sector members that communicate via one hop to the SH (see Figure 3c), where $S H_{i} \in[1 . . S]$. Each node is configured to be aware of the deployment layout by knowing: (1) each $\mathrm{SH}$ is assigned with the sector number as a virtual address and node id; and (2) all member nodes know their own node id and number of tracks $(m)$ and sectors $(n)$ of the network field. As shown in Figure 3b, the intra-sector communication (i.e., communication from sector members to $\mathrm{SH}$ or vice-versa) is constrained to one hop while inter-sector transmission is multi-hop. For simplification, the sensor nodes inside each sector are not shown explicitly in Figure 3b. Instead, an aggregated link (see Figure 3c) is shown to represent the total traffic from member nodes to head node.
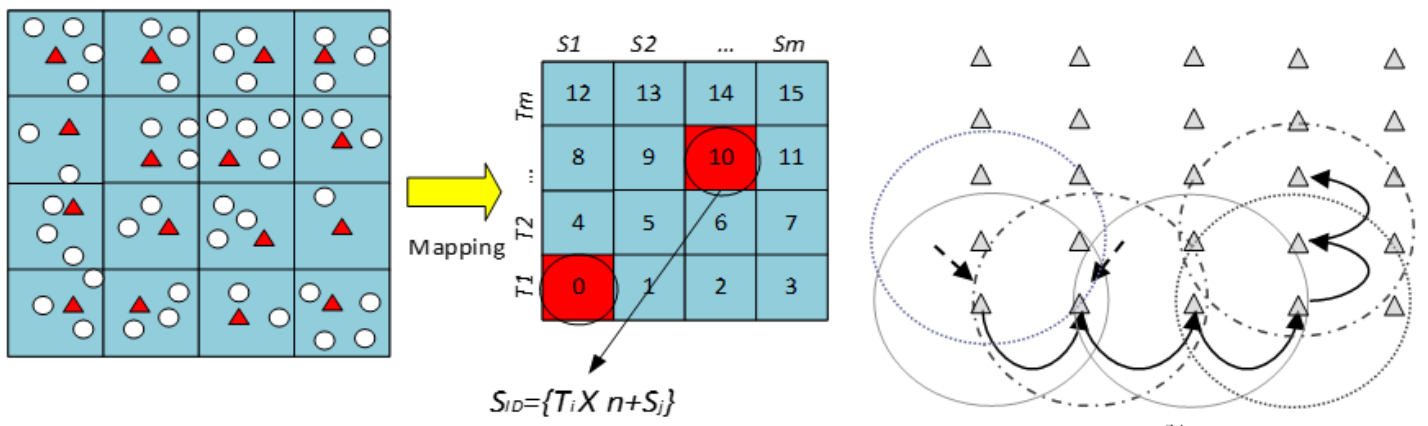

(a)
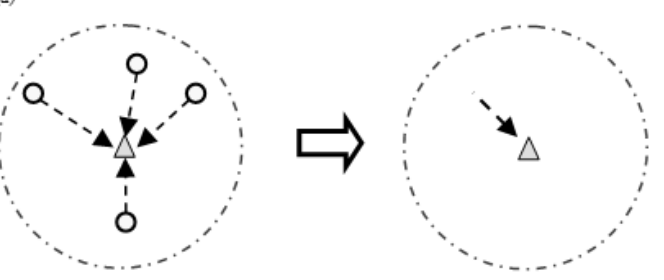

(b)

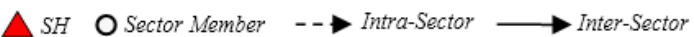

Figure 3. (a) DBDCS Mapping; (b) inter-sector communication; and (c) intra-sector member node to head node communication

\subsection{Skyline Definition with Metric-Based Searching}

Metric space $M$ can be defined as a pair $M=(D, d)$, where $D$ is the domain of objects and $d$ is the distance function $-d: D \times D \rightarrow R$ satisfying the following constraints (Equation (1)) for all objects $a, b, c \in D:$

$$
\begin{array}{cl}
d(a, b) \geqslant 0 & \text { (non - negativity) } \\
d(a, b)=0 & \text { (identity) } \\
d(a, b)=d(b, a) & \text { (symmetry) } \\
d(a, c) \leqslant d(a, b)+d(b, c) & \text { (triangle inequality) }
\end{array}
$$

In this metric space, considering that smaller values are preferable to larger ones for a set of l-dimensional data, the dynamic skyline query result set can be defined as: 
$\operatorname{Skyline}(q, r):$

$$
X=\left\{\begin{array}{l}
\forall_{i} \in[1, l], a, b \in I \mid a(i) \leqslant b(i) \text { and } \\
\exists_{i} \in[1, l], a(i)<b(i) \text { and } \\
d(q, a) \leqslant r
\end{array}\right\}
$$

In Equation (2), $q$ denotes the query point, $a(i)$ denotes the value of the $i t h$ attribute of an object and $r$ defines the range of the region of interest. The data space is divided into $S$ sectors with a pivot point, denoted by $P_{i}$, for each sector $S_{i}$. The iDistance key for an object $x \in D$ can be defined as (Figure 4a, Equation (3)):

$$
i \operatorname{Dist}(x)=d\left(P_{i}, x\right)+i \cdot c
$$

In Equation (3), $c$ is the separating constant for individual sectors. Given $q \in D$, the region of interest with radius $r$ can be defined as (Figure $4 b$, Equation (4)):

$$
r=\left[d\left(P_{i}, q\right)+i \cdot c-r, d\left(P_{i}, q\right)+i \cdot c+r\right]
$$

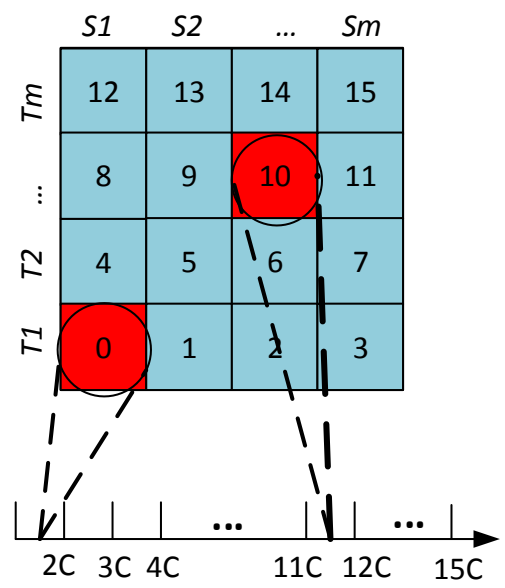

(a)

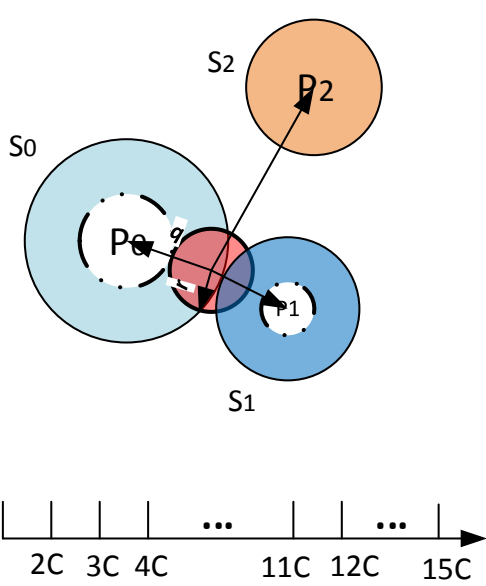

(b)

Figure 4. (a) Data mapping; and (b) range query example.

\subsection{Data Processing and Mapping}

A sensed event $E$ can be defined by an $l$-dimensional tuple, $\left(A_{1}, A_{2}, A_{3}, \ldots A_{l}\right)$ where $A_{g}, \forall_{g} \in[1, l]$ denotes the $g$ th attribute and $D_{A g}$ is the domain of attribute $A_{g}$. Each member node of a sector transmits the sensed event as an $l$-tuple $\left\langle v_{i 1}, v_{i 2}, \ldots \ldots, v_{i l}\right\rangle_{k}$, where $1 \leqslant i \leqslant M_{k}, M_{k}$ is the total number of member nodes in $k t h$ sector and $v_{i j}$ denotes the value of the $j t h$ attribute received from ith node of $k t h$ sector. A $S H$ node after collecting tuples from all the member nodes aggregates them at the end of each epoch before finding the mapping for the target $S H$.

Hence, after aggregation at epoch $t$

$$
\begin{gathered}
\mathrm{E}_{\mathrm{k}}(\operatorname{Agg}(t))=\int_{\operatorname{Agg}_{\mathrm{i}=1}}^{M_{k}}\left\langle v_{i 1}, v_{i 2}, \ldots . ., v_{i l}\right\rangle(t) \\
=\left\langle\psi_{1}, \psi_{2}, \ldots \ldots, \psi_{l}\right\rangle(t)
\end{gathered}
$$

Here,

$$
\psi_{j}=\left\{\max _{i=1}^{M_{k}} v_{i j}, \min _{i=1}^{M_{k}} v_{i j}, a v g_{i=1}^{M_{k}}\right\}, j \in[1, l], k \in[1, S]
$$


In Equation (6), $M_{k}$ denotes the number of member nodes in $k t h$ sector. It is assumed that all attribute's aggregated values of $\psi_{i}$ have been normalized to be between 0 and 1 . As shown in Table 1 , weights have been assigned to different attributes based on their importance in the event description. Hence, an attribute with higher weight has higher influence in deciding the similarity among events.

Table 1. Weight settings.

\begin{tabular}{cc}
\hline Attribute & Weight \\
\hline$A_{1}$ & $w_{1}$ \\
$A_{2}$ & $w_{2}$ \\
$\ldots$. & $\ldots$. \\
$A_{l}$ & $w_{l}$ \\
\hline
\end{tabular}

\subsubsection{Pivot Point Generation}

$$
\begin{gathered}
\alpha_{\min }=\sum_{i=1}^{l}\left(\left(A_{i(\min )} / A_{i(\max )}\right) \times w_{i}\right) \\
\alpha_{\max }=\sum_{i=1}^{l}\left(\left(A_{i(\max )} / A_{i(\max )}\right) \times w_{i}\right) \\
\beta=\sum_{i=1}^{l}\left(\left(A_{i(\text { avg })} / A_{i(\max )}\right) \times w_{i}\right) \\
\delta=\sum_{i=1}^{l}\left(\left(A_{i(\theta)} / A_{i(\max )}\right) \times w_{i}\right)
\end{gathered}
$$

In Equations (7.1)-(7.4), $\mathrm{A}_{\mathrm{i}(\min )}, \mathrm{A}_{\mathrm{i}(\max )}, \mathrm{A}_{\mathrm{i}(\text { avg })}$ and $\mathrm{A}_{i(\theta)}$ denote the minimum, maximum, average and threshold value of $i$ th attribute. Based on Equations (7.1) and (7.2), the domain of the hash key, denoted by $H_{D}$, is $\alpha$ ( $\left.\alpha_{\min }, \alpha_{\max }\right)$. The center of gravity, denoted by $\beta$, is derived in Equation (7.3) to find the normalized center point of the domain of the hash key $H_{D}$, whereas $\delta$ is the separating factor between two pivot points. However, in order to balance the load among sectors, it is important to find the range where the concentration of the data points is high. Hence, $\beta$ and $\delta$ can be used to find the range for center of gravity, denoted by $\beta\left(\beta_{\text {range-min }}, \beta_{\text {range-max }}\right)$, as shown in Equation (8).

$$
\begin{aligned}
& \beta_{\text {range }-\min }=\beta-\delta \\
& \beta_{\text {range }- \text { max }}=\beta+\delta
\end{aligned}
$$

Thus, the separating step, denoted by $\eta$, between two pivot points in COM range can be defined by:

$$
\eta=\left(\beta_{\text {range }-\max }-\beta_{\text {range }-\min }\right) / S-1
$$

Thus, the pivot points for $S$ sectors can be defined in each sector head by:

$$
\mathrm{P}_{i}= \begin{cases}\alpha_{\min }, & i=0 \\ \beta_{\text {range }-\min }+i \times \eta, & 0<i<S \\ \alpha_{\max }, & i=S\end{cases}
$$



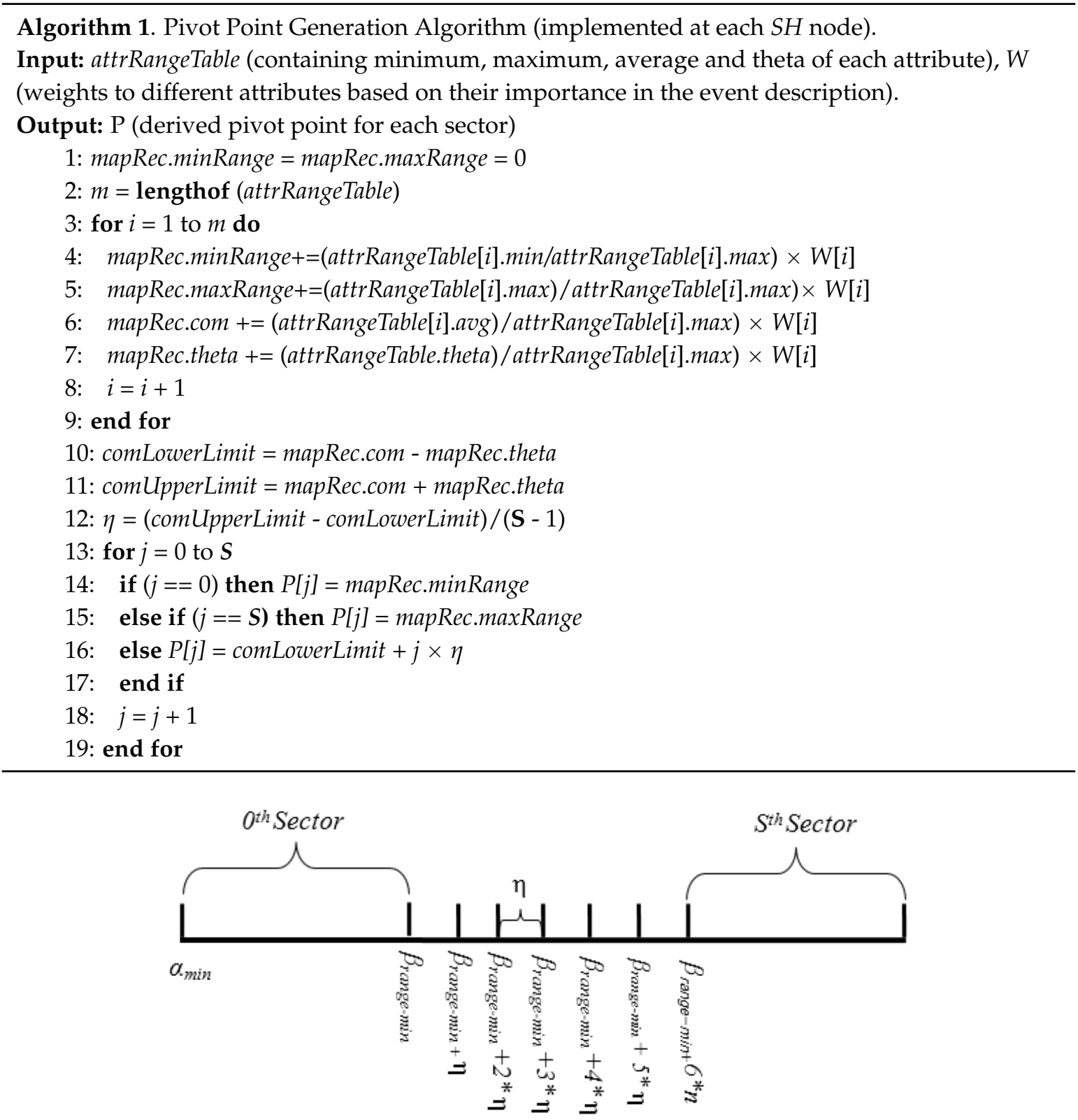

Figure 5. Pivot point generation example.

Algorithm 1 uses Equations (8)-(10) for calculating pivot points. Figure 5 illustrates the domain and sub-domain of pivot points.

\subsubsection{Mapping}

Given $l$ attributes in an attribute list associated with weight $w_{j}(1 \leqslant j \leqslant l)$ in a WSN application, the source $\mathrm{SH}_{k}$ generates the hash value by:

$$
h=\sum_{j=1}^{l}\left(\left(a v g_{i=1}^{M_{k}} v_{i j} / A_{j(\max )}\right) \times w_{j}\right)
$$

Hence, after each epoch, $S H_{k}$ forwards the aggregated event $E_{k}=\left\langle\left[\psi_{1}, \psi_{2}, \ldots, \psi_{l}\right],[t, h]\right\rangle$, where $t$ denotes the epoch number, to the destination sector head denoted by $S H_{i}$ where, $P_{i} \leqslant h<P_{i+1}$ and $P_{i}$ and $P_{i+1}$ is the lower and upper limit of $i$ th sub-interval, respectively. 


\subsection{Insertion}

Within a sector, data are further distributed among nodes according to their distance from the $\mathrm{SH}$. In order to do so, a sector is divided into segments. Figures 6 and 7 and Table 2 illustrate the idea of sector segmentation. Given a $k t h$ sector containing $M_{k}$ member nodes, the $S H_{k}$ first sorts all member nodes based on RSSI in ascending order. The member nodes are then divided into $r$ segments. Each segment forms a circle, denoted by $B_{(X, Y)}\left(r_{i}\right)$, where the center of the circle is $(X, Y)$ with radius $r_{i} .(\mathrm{X}, \mathrm{Y})$ is the geographic co-ordinates for $S H_{k}$. The number of segments depends on the WSN application, sector size and the number of member nodes in each sector. Thus the set of sensors that are within a Euclidean distance $r_{i}$ from $(X, Y)$ form the segment defined by:

$$
\begin{aligned}
& B_{(X, Y)}\left(r_{i}\right)=\left\{\text { SensorsCoordinate }(x, y):|(X, Y),(x, y)| \leqslant r_{i}\right\} \\
& \beta_{k}=\left(\mathrm{P}_{k+1}-\mathrm{P}_{k}\right) / r, \quad 1 \leqslant k \leqslant S \\
& \left\{\mathrm{P}_{M_{K}(i)}\right\}_{k=1}^{S}=\left\{\begin{array}{lc}
\mathrm{P}_{k}, & i=0 \\
\mathrm{P}_{k}+\beta \times i, & 0<i<r \\
\mathrm{P}_{k+1}, & i=r
\end{array}\right.
\end{aligned}
$$

By Equations (13) and (14), the pivot points of $r$ segments within the $k t h$ sector are calculated. An event with hash value, denoted by $h$, is stored in a member sensor node of ith segment where $\mathrm{P}_{M_{K}(i)} \leqslant h \leqslant \mathrm{P}_{M_{K}(i+1)}$. In order to balance the load, data are distributed among the nodes inside a segment in a round robin fashion (see Algorithm 2).
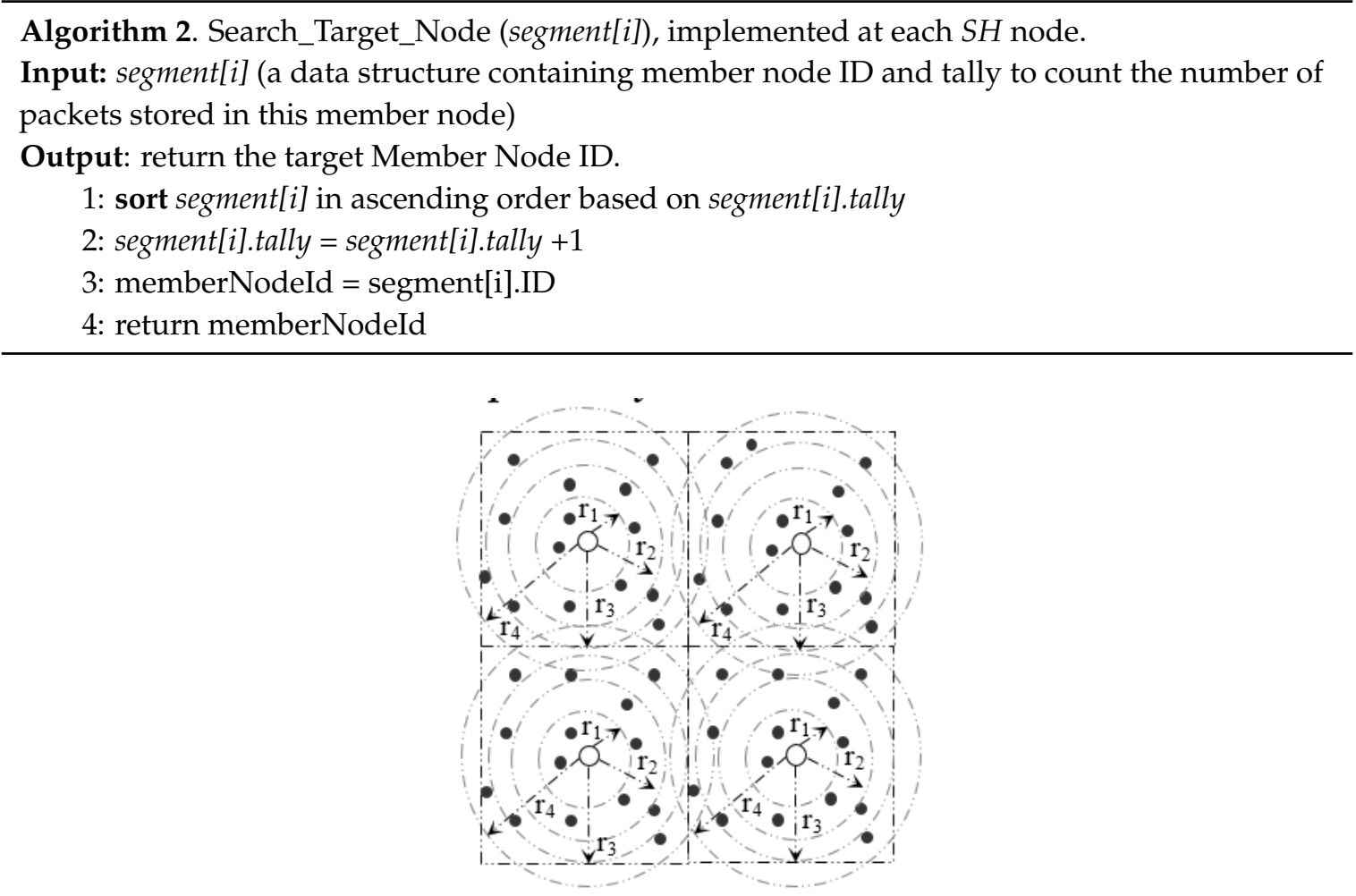

Figure 6. Formation of Segments inside a Sector. 


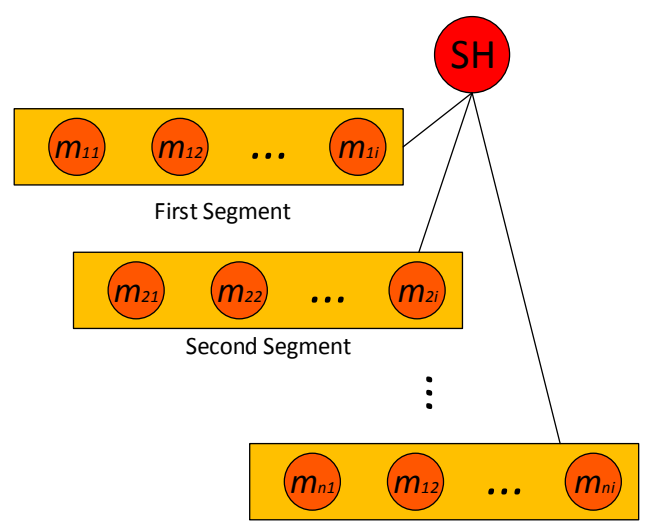

$r_{\text {th }}$ Segment

Figure 7. Segmentation architecture of member nodes inside a sector.

Table 2. Member table of a SH node.

\begin{tabular}{|c|c|}
\hline Member Node Id & $\begin{array}{c}\text { Received Signal } \\
\text { Strength Indicator }\end{array}$ \\
\hline$m_{1}$ & $R^{\prime} S S I_{1}$ \\
\hline$m_{2}$ & $\mathrm{RSSI}_{2}$ \\
\hline$m_{3}$ & $\mathrm{RSSI}_{3}$ \\
\hline$m_{4}$ & $\mathrm{RSSI}_{4}$ \\
\hline.$\cdot$ &.$\cdot$ \\
\hline$\ddot{m_{i}}$ & $R \ddot{S S I} I_{i}$ \\
\hline.$\cdot$ &.. \\
\hline .. & .. \\
\hline.. &.. \\
\hline$\ddot{M}_{K}$ & $\ddot{R S S I_{k}}$ \\
\hline
\end{tabular}

\subsection{Skyline Query}

A threshold based hierarchical approach has been used in this research to calculate $D S(q, r)$. The threshold based hierarchical approach uses the temporal correlation among the sectors and segments of a sector. All the key notations used in this section are listed in the Table 3 to increase reader comfort.

Table 3. Skyline Notation.

\begin{tabular}{|c|c|}
\hline Symbol & Description \\
\hline$e_{i}$ & An event at $S H_{i}$ or segment $r_{i}$ of a sector \\
\hline$E$ & A set of events \\
\hline$\theta_{i}$ & The threshold point of $S H_{i}$ or Segment $r_{\mathrm{i}}$ \\
\hline$L D S_{i}$ & The local dynamic skyline of $S H_{i}$ or Segment $r_{i}$ \\
\hline$e_{i} \prec_{(q, r)} e_{j}$ & $\begin{array}{l}\text { Event } e_{j} \text { is dominated by event } e_{i} \text { with respect to query point } q \text { and the target } \\
\text { region of interest is a circle of radius } r \text { centered at } q .\end{array}$ \\
\hline$e_{i} \preccurlyeq_{(q, r)} e_{j}$ & $\begin{array}{c}\text { Event } e_{j} \text { is dominated by or equal to event } e_{i} \text { with respect to query point } q \text { and } \\
\text { the target region of interest is a circle of radius } r \text { centered at } q .\end{array}$ \\
\hline$e_{i}<_{(q, r)} E$ & $\begin{array}{c}\text { Each event in } E \text { is dominated by event } e_{i} \text { with respect to query point } q \text { and the } \\
\text { target region of interest is a circle of radius } r \text { centered at } q \text {. }\end{array}$ \\
\hline$e_{i} \preccurlyeq_{(q, r)} E$ & $\begin{array}{l}\text { Each event in } E \text { is dominated by or equal to event } e_{i} \text { with respect to query } \\
\text { point } q \text { and the target region of interest is a circle of radius } r \text { centered at } q \text {. }\end{array}$ \\
\hline
\end{tabular}




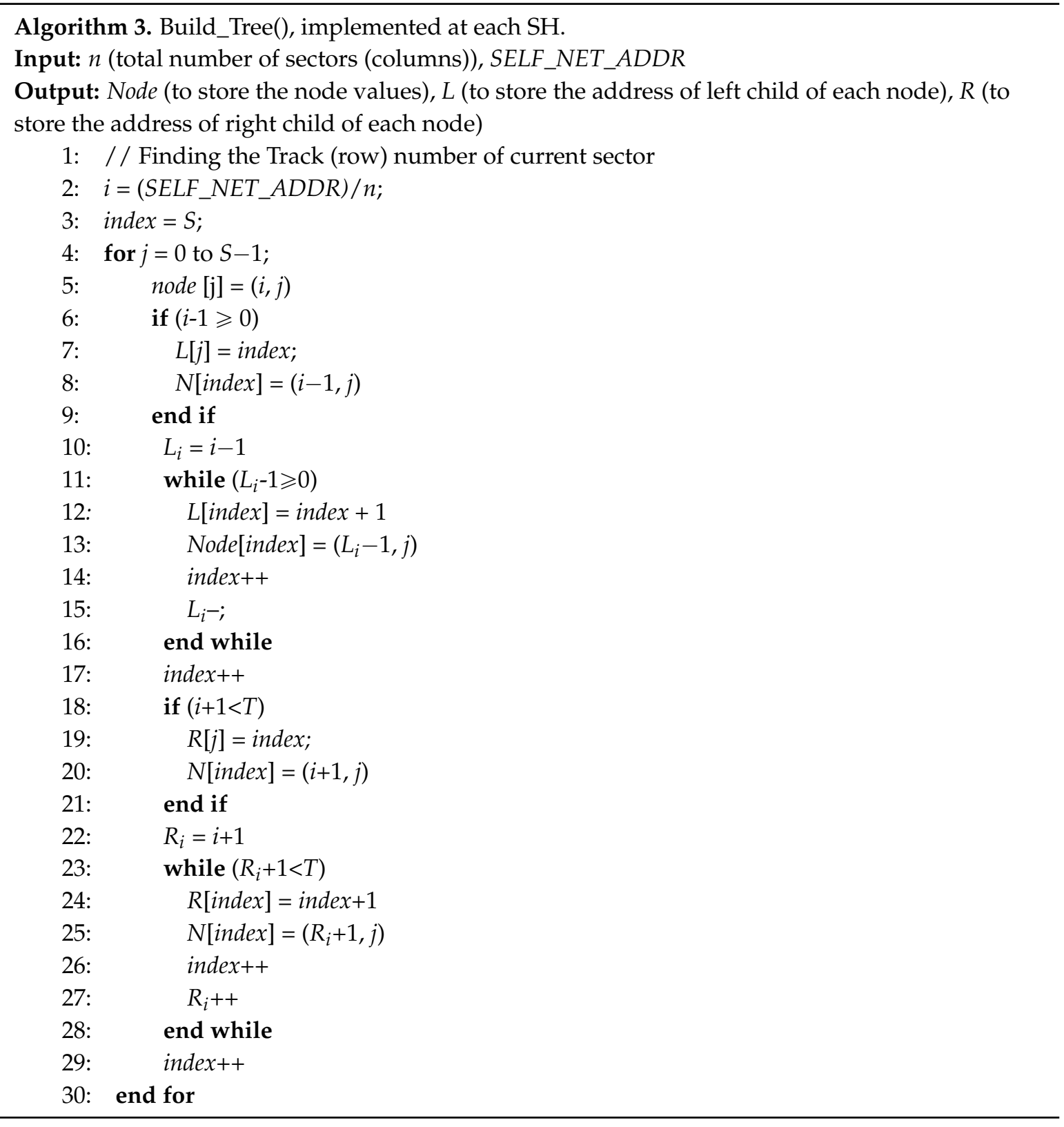

\subsubsection{Tree Structure Construction}

Each SH constructs a tree considering itself as the root using Algorithm 3. Figures 8 and 9 illustrate an example of the formation of a tree rooted at the 13th $(2,3)$ Sector. Sector $(2,0),(2,1),(2,2),(2,3)$, $(2,4)$ and $(2,5)$ are the child nodes of Sector $(2,3)$. The sectors lying at the same column but upper row and lower row of each of the child nodes of root node are added to the left and right branch, respectively. For example, Sector $(1,0),(0,0)$ and $(3,0),(4,0)$ are added to the left and right branch, respectively, of Sector $(2,0)$. The hierarchy is further maintained among segments inside each sector, as shown in Figure 10. 


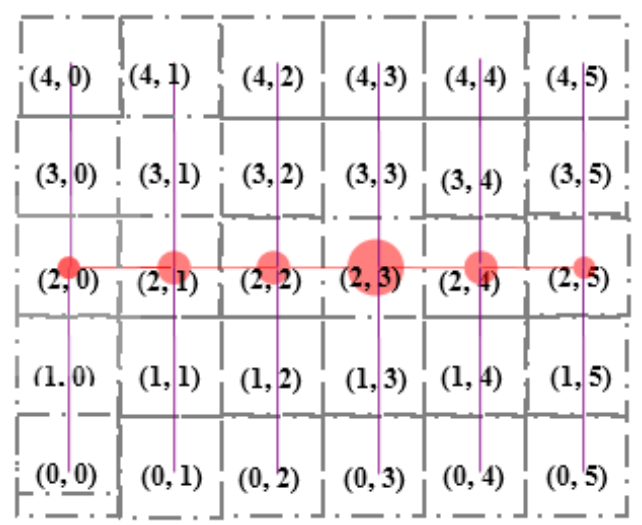

Figure 8. SH of 13 th Sector implies Algorithm 3 in order to convert the $5 \times 5$ grid into a tree rooted at 13 th $(2,3)$ Sector.

$(2,3)$

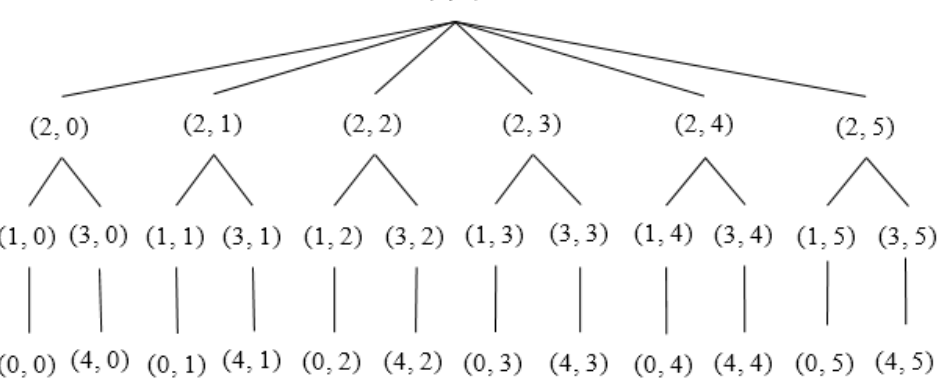

Figure 9. Tree rooted at 13 th Sector $(2,3)$.

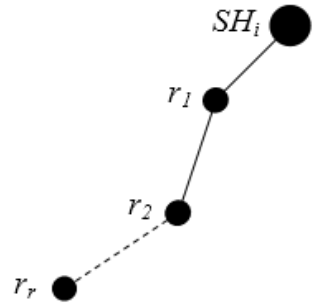

Figure 10. Hierarchy based on segmentation.

\subsubsection{Basic System Operation}

A query node first calculates hash $h_{q}$ using Equation (10) for the Dynamic Skyline Query (DS $(q, r))$. The query is then forwarded to the $S H_{i}$ where $P_{i} \leqslant h_{q} \leqslant P_{i+1} . S H_{i}$ finds the range of the query, i.e., $\left[h_{q}-r, h_{q}+r\right]$. Hence, the target sectors where the sample dataset of the query need to be considered are $S H_{j}, S H_{j+1}, \ldots, S H_{k}$, here $P_{j} \leqslant h_{q-r} \leqslant P_{j+1}, P_{k} \leqslant h_{q+r} \leqslant P_{k+1}$ and $j \leqslant k$. The threshold based hierarchical approach includes three phases-Tree Propagation, Regular Update and Triggered Query.

$\mathrm{SH}_{i}$ issues a Triggered Query containing $\mathrm{LS}_{i}$ to $\mathrm{SH}_{j+1}, \mathrm{SH}_{j+2} \ldots, \mathrm{SH}_{k}$. The Triggered Query is issued to ensure fetching all possible events that might be included in the final skyline but was not reported during the Regular Update phase. $S H_{i}$ sends Triggered Query to each of its child $S H_{j}$ that satisfies $L S_{i} \npreceq \theta_{j}$. Any child node $S H_{j}$ satisfying $L S_{i} \leq \theta_{j}$ can be discarded since no ineligible event can exist in the final skytline. There cannot exist any event that can be eligible to be included in the final skyline. An internal child node $S H_{j}$ after receiving Triggered Query $L S_{p}$ from its parent computes $L S_{j}$ among $L S_{p}$ and its non-reported points. $S H_{j}$ then forwards $L S_{j}$ to each of its children $S H_{k}$ that satisfies $L S_{j} \npreceq \theta_{k}$ and waits for a reply with new points that are not dominated by $L S_{j} . S H_{j}$ updates the local skyline after receiving replies from all of its child nodes and finally replies to its parent $S H_{p}$ with the new 
skyline event set. In contrast, a leaf node $S H_{j}$ reports nothing if it reports $e_{j}$ in the first phase or satisfies $L S_{i} \leq \theta_{j}$. Otherwise, it reports event $e_{j}$.

Figure 11 illustrates the functionality of the two phases-Regular Update and Triggered Query in a particular scenario. In the first phase (Regular Update) (Figure 11a), $\mathrm{SH}_{0}$ and $\mathrm{SH}_{7}$ reports $e_{0}$ and $e_{7}$ to $\mathrm{SH}_{3}$ and $\mathrm{SH}_{4}$, respectively. However, $e_{7}$ and $e_{3}$ have been pruned by $e_{4}$. Thus, the skyline set after the first phase includes $\left\{e_{0}, e_{4}\right\}$ (see Figure 11b). Figure 11c,d illustrates the second phase. $\mathrm{SH}_{4}$ issues a Triggered Query containing $\left\{e_{0}, e_{4}\right\}$ to $\mathrm{SH}_{3}, \mathrm{SH}_{6}, \mathrm{SH}_{5}$, and $\mathrm{SH}_{8}$ (here, it is assumed that $\left[h_{q}-r, h_{q}+r\right]$ covers all the tree $\mathrm{SH}$ ). $\mathrm{SH}_{0}, \mathrm{SH}_{1}, \mathrm{SH}_{7}$ and $\mathrm{SH}_{2}$, however, are discarded since their corresponding thresholds are dominated by $e_{4}$. After receiving the Triggered Query, $\mathrm{SH}_{5}$ prunes $e_{5}$ and forwards the updated skyline set $\left\{e_{0}, e_{4}\right\}$ to $\mathrm{SH}_{8} . \mathrm{SH}_{8}$ calculates its local skyline and prunes among the query it receives from its parent and its own event $e_{8}$. Since $e_{8}$ is not dominated, it has also been included into the final local skyline set, i.e., $\left\{e_{0}, e_{4}, e_{8}\right\}$, and is finally sent back to the $S H_{4}$. It is to be noted that, during both phases, $\mathrm{SH}_{1}$ and $\mathrm{SH}_{2}$ do not need to transmit any event.

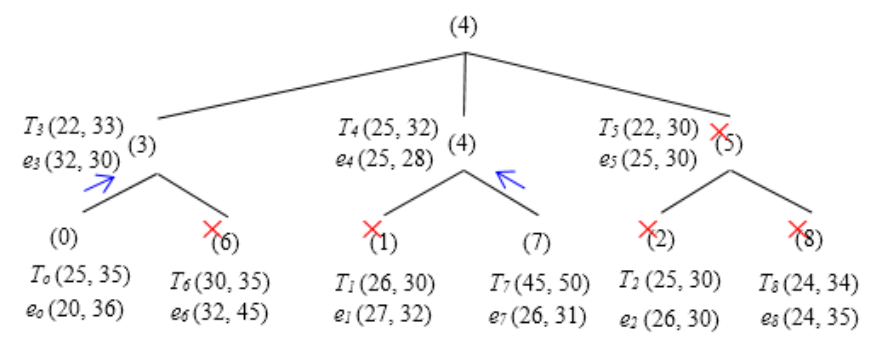

(a)

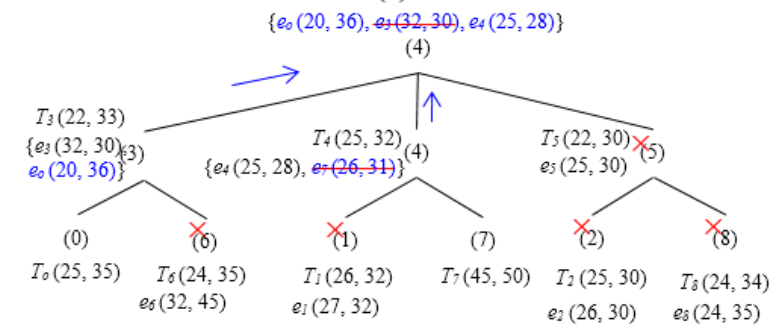

(b)

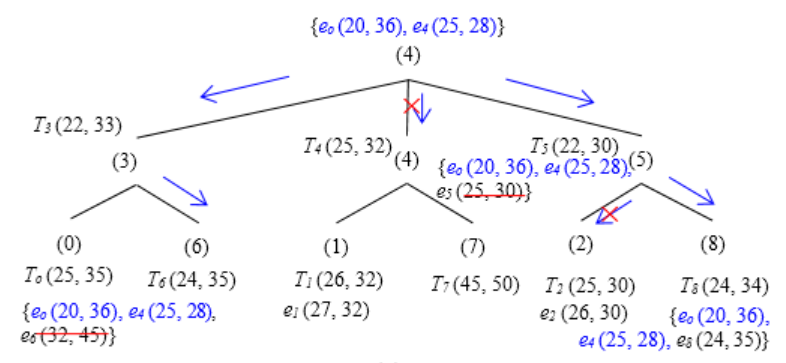

(c)

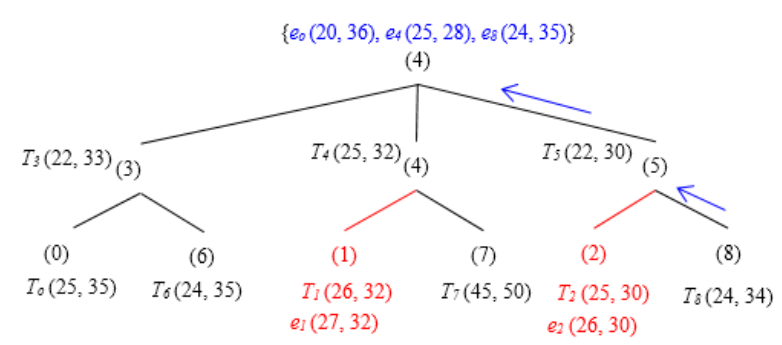

(d)

Figure 11. Basic Approach Example ( $3 \times 3$ Grids). (a) Regular update. (b) Skyline set after first phase. (c) Triggered query. (d) Skyline set after second phase. 


\section{Performance Evaluation}

Simulations were conducted using Castalia v3.2 [29] running on top of OMNET++ [31] to evaluate the performance of EDDS. The system parameters and their settings used in the experiments are summarized in Table 4. The network model (illustrated in Section 3.1) was tested in four rectangular fields with different parameter settings. Sensor MAC (SMAC) [32] and Sector Based Distance Routing (SBD) [15] are used in MAC and routing layer. Simulations were run 30 40 times with varying-channel affecting seeds to provide results that included average and 95\% confidence interval. In Section 4.1, possible distribution of data throughout the network is presented. Section 4.2 evaluated EDDS in terms of energy consumption, latency and accuracy in a network of 180 nodes in a $90 \mathrm{~m} \times 90 \mathrm{~m}$ $\left(8100 \mathrm{~m}^{2}\right)$ rectangular field. In Section 4.3 , the performance of EDDS was tested using four different rectangular fields with four different distributions respectively. In Section 4.4, the performance of EDDS is evaluated against SkySensor in terms of data loss, data uniformity, success rate and resilience to node failure.

\subsection{Data Distribution}

Figure 12 exploits different possible data dispersal with the realization of the mapping algorithm illustrated in Section 3.2. This experiment was conducted in a network of 80 nodes in a $60 \mathrm{~m} \times 60 \mathrm{~m}$ $\left(3600 \mathrm{~m}^{2}\right.$, total number of sectors is $\left.16(4 \times 4)\right)$ field with a $60 \mathrm{~min}$ simulation time. The data production rate per sector was five packets per second. Figure 12a shows uniform distribution, which means data are stored uniformly among all sectors of the network. Figure $12 \mathrm{~b}$ shows a central distribution in which data are usually located among central sector of the network. Figure 12c,d represents the lower bound distribution and upper bound distribution, where data are stored among lower and upper sectors of the network, respectively.

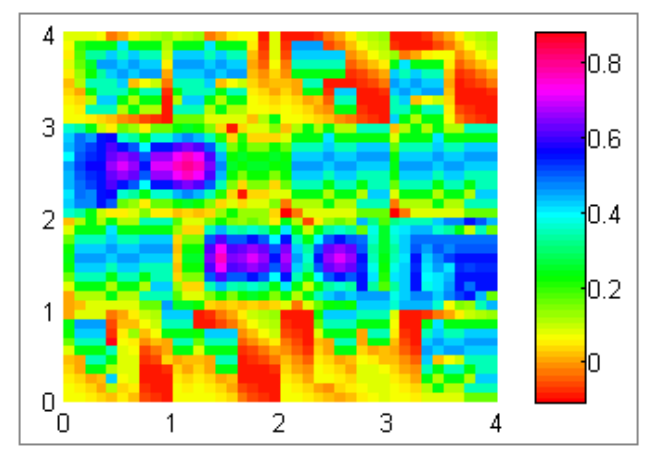

(a)

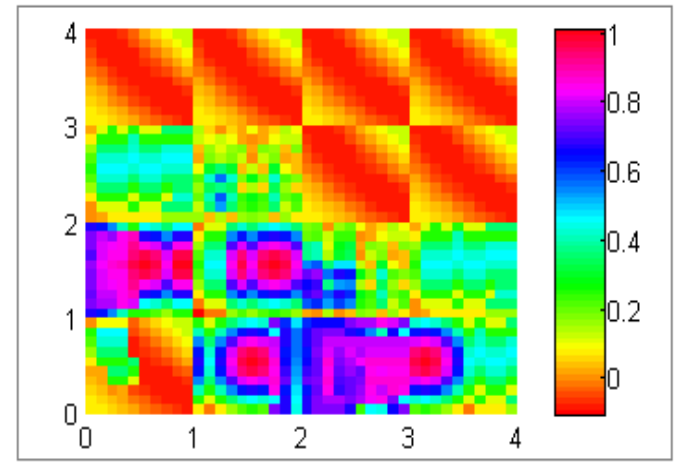

(c)

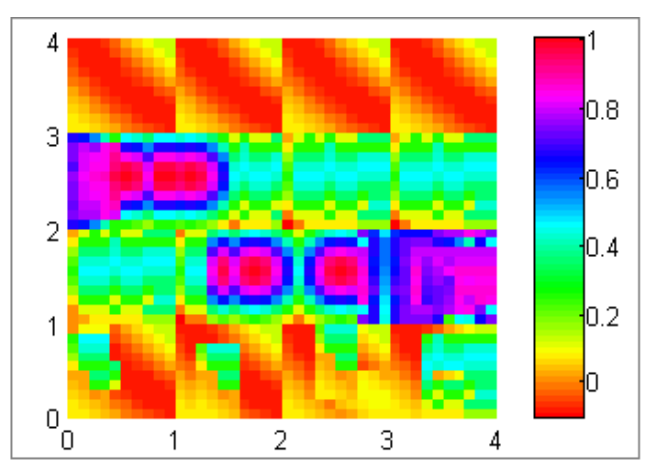

(b)

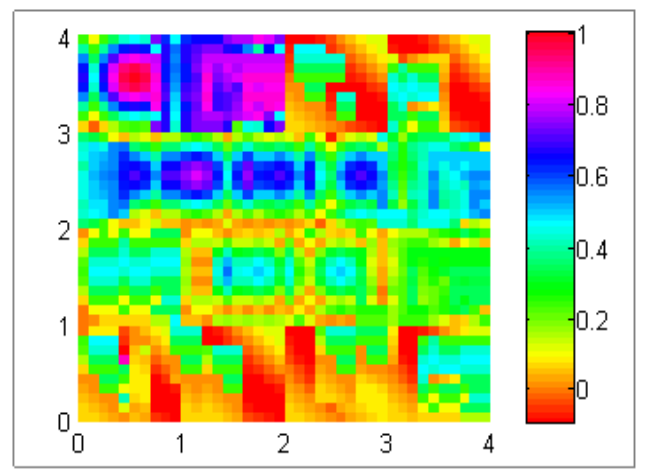

(d)

Figure 12. (a) Uniform distribution; (b) central distribution; (c) lower bound distribution; and (d) upper bound distribution. 


\subsection{Efficiency}

The efficiency of the proposed approach is measured based on three parameters-energy consumption, latency and accuracy. These performance metrics and scalability of the proposed model is directly affected by the query range, which varies based on the average number of sectors indicated by $\alpha$. With the increase of $\alpha$, the number of messages that are generated due to three fundamental operations such as Tree Propagation, Regular Update and Triggered Query increases exponentially. Thus, in a model such as skyline query, it is an utmost challenge to minimize the energy consumption and latency while maximizing the accuracy. This experiment was conducted in a $90 \mathrm{~m} \times 90 \mathrm{~m}$ rectangular field, in which 180 nodes were randomly and independently disseminated. The data distribution used in this experiment was uniform. The query rate varied from 0.1 to 0.5 queries per sector per second and the simulation was run for $60 \mathrm{~min}$. The Skyline Query overhead is comparatively high due to its three phase query calculation. Figure 13a-c presents the average energy consumption (J) per node, latency (s) and accuracy (accuracy was defined as the percentage of skyline queries that were correctly resolved) as a function of the query rate per sector per second. In this experiment, the query range was varied in four different ways as shown in Figure 13. In order to show the scalability of the system, $\alpha$ has been varied exponentially up to 36 , where 36 is the total number of sectors in a $90 \mathrm{~m} \times 90 \mathrm{~m}$ (Table 4) rectangular field. From Figure 13a,b, it is noted that the overhead in terms of energy consumption and latency grows radically as $\alpha$ increases. It is also to be noted that the data in Figure 13a are presented in logarithmic scale in order to reduce the wide range to a more manageable size. However, the accuracy of the query response was very high (see Figure 13c) and close to $100 \%$. Packet loss due to interference causes some minor inaccuracy.

Table 4. Simulation Parameters.

\begin{tabular}{|c|c|}
\hline Parameter & Setting \\
\hline Field Size $(F)$ & $60 \times 60 \mathrm{~m}^{2}, 90 \times 90 \mathrm{~m}^{2}, 120 \times 120 \mathrm{~m}^{2}, 150 \times 150 \mathrm{~m}^{2}$ \\
\hline Number of Nodes $(N)$ & $\begin{array}{c}80\left(3600 \mathrm{~m}^{2}\right), 180\left(8100 \mathrm{~m}^{2}\right), 320\left(14,400 \mathrm{~m}^{2}\right) \\
500\left(22,500 \mathrm{~m}^{2}\right)\end{array}$ \\
\hline Number of Sectors/Field Size $(S / F)$ & $\begin{array}{c}16 /\left(60 \times 60 \mathrm{~m}^{2}\right), 36 /\left(90 \times 90 \mathrm{~m}^{2}\right), 64 /\left(120 \times 120 \mathrm{~m}^{2}\right) \\
100 /\left(150 \times 150 \mathrm{~m}^{2}\right)\end{array}$ \\
\hline Member Node Density $\left(f_{m}\right)$ & 1 node $/ 56.25 \mathrm{~m}^{2}$ \\
\hline Sector Head Node $(\mathrm{SH})$ Density $\left(f_{S H}\right)$ & 1 node $/ 225 \mathrm{~m}^{2}$ \\
\hline Radio Range (member node) & $\sim 8 \mathrm{~m}$ \\
\hline Radio Range $(\mathrm{SH})$ & $\sim 20 \mathrm{~m}$ \\
\hline Transmission Power & $0 \mathrm{dBm}(\mathrm{SH}),-5 \mathrm{dBm}$ (member node) \\
\hline $\begin{array}{c}\text { Power Consumption in Sending and } \\
\text { Receiving Messages }\end{array}$ & $57.42 \mathrm{~mW}(\mathrm{SH}), 46.2 \mathrm{~mW}$ (member node) \\
\hline Power Consumption Per Sensing & 0.02 mJoule \\
\hline $\begin{array}{c}\text { Data Rate, Modulation Type, Bits Per Symbol, } \\
\text { Bandwidth, Noise Bandwidth, Noise } \\
\text { Floor, Sensitivity }\end{array}$ & $\begin{array}{c}250 \mathrm{Kbps}, \mathrm{PSK}, 4,20 \mathrm{MHz}, 194 \mathrm{MHz},-100 \mathrm{dBm}, \\
-95 \mathrm{dBm}\end{array}$ \\
\hline pathLossExponent & 2.4 \\
\hline Initial Average Path Loss $\left(P L\left(d_{0}\right)\right)$ & 55 \\
\hline Reference Distance $\left(d_{0}\right)$ & $1.0 \mathrm{~m}$ \\
\hline Gaussian Zero-Mean Random Variable $\left(X_{\alpha}\right)$ & 4.0 \\
\hline Routing Protocol & SBD [15] \\
\hline MAC Protocol, Maximum Transimission Retries & SMAC [32], 2 \\
\hline $\begin{array}{c}\text { SMAC Acknowledgment, Synchronization, RTS, CTS } \\
\text { Packet Size }\end{array}$ & $11,11,13,13$ bytes \\
\hline
\end{tabular}




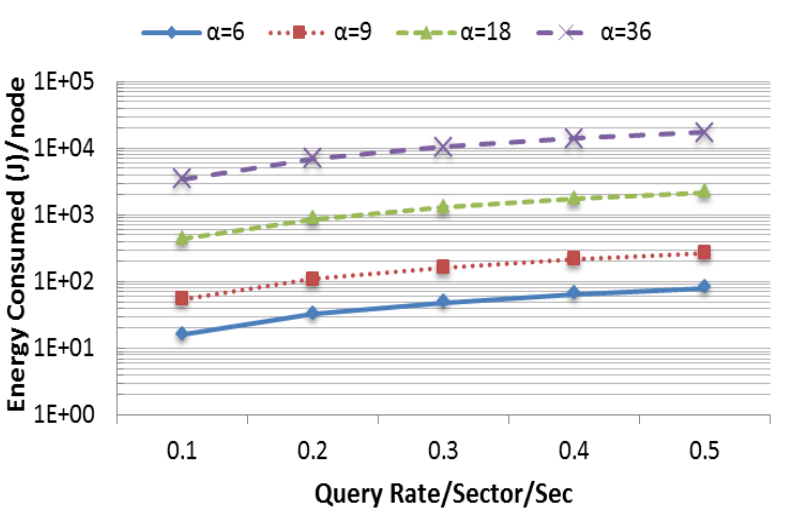

(a)

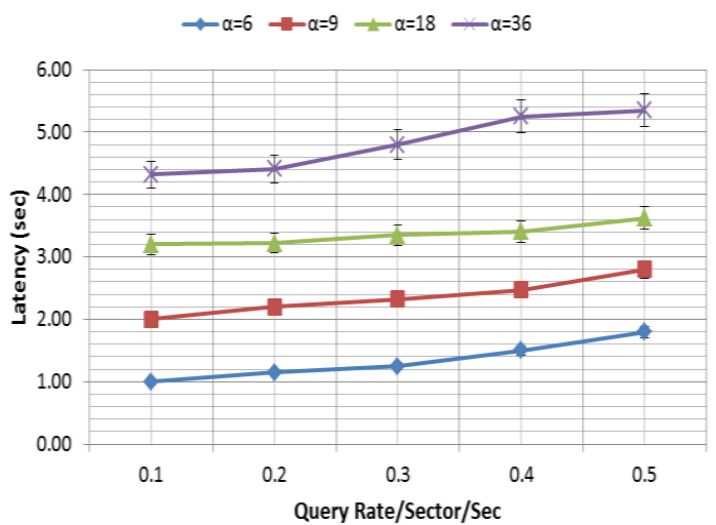

(b)

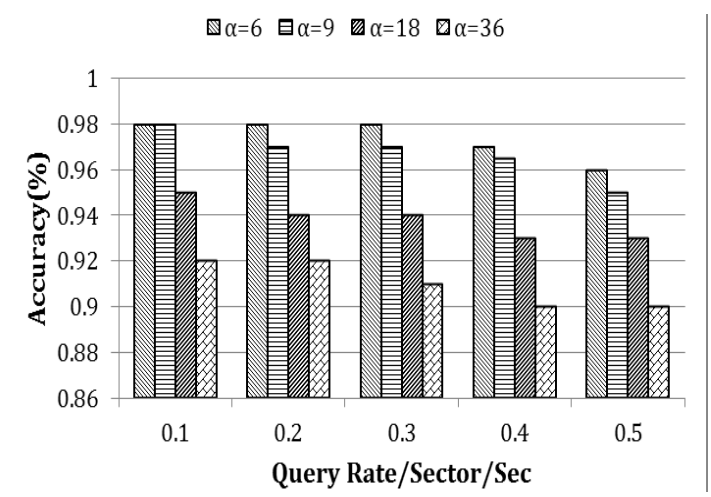

(c)

Figure 13. (a) Energy consumption; (b) latency; and (c) accuracy.

\subsection{Robustness}

In this section, robustness of EDDS was evaluated through two different experiments with the variation of four different network sizes. In the first experiment, energy consumption, latency, and accuracy were measured for varying the query rate, while, in the second experiment, they were measured for four different data distributions.

\subsubsection{Network Size}

Experiments were carried out by varying the network field sizes such as $60 \times 60 \mathrm{~m}^{2}, 90 \times 90 \mathrm{~m}^{2}$, $120 \times 120 \mathrm{~m}^{2}$ and $150 \times 150 \mathrm{~m}^{2}$ containing 80,180, 320 and 500 nodes, respectively. The size of the region of interest was kept fixed, i.e., the value of $\alpha$ was 9. The rate of the query was varied from 0.1 to 0.5 queries per sector per second. In Figure 14, the rate of the query is represented by $\beta$. Figure $14 a, b$ demonstrates that energy consumption and latency is exponentially proportional to the value of $\beta$. However, when $\beta$ is constant, they grow linearly with the size of the network. Figure $14 \mathrm{c}$ shows the percentage of accuracy as a function of network size. The accuracy drops slightly with the scale of the network. 


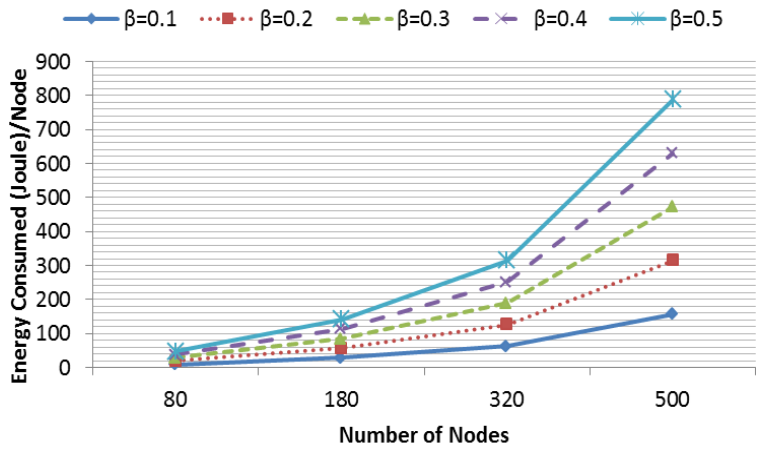

(a)

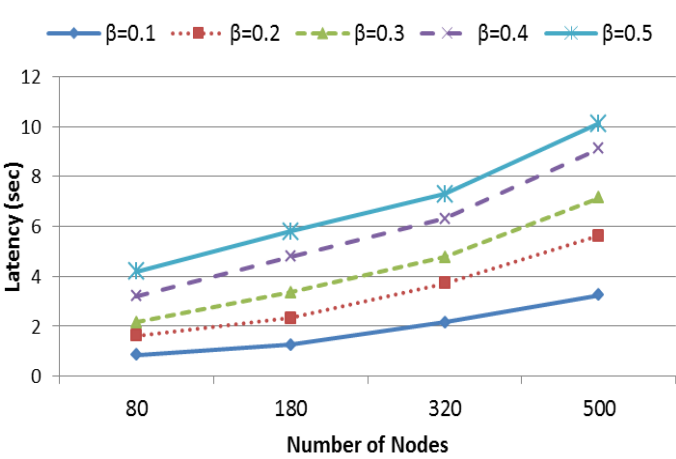

(b)

$\square \beta=0.1 \quad \boxminus \beta=0.2 \quad \square \beta=0.3 \quad \square \beta=0.4 \square \beta=0.5$

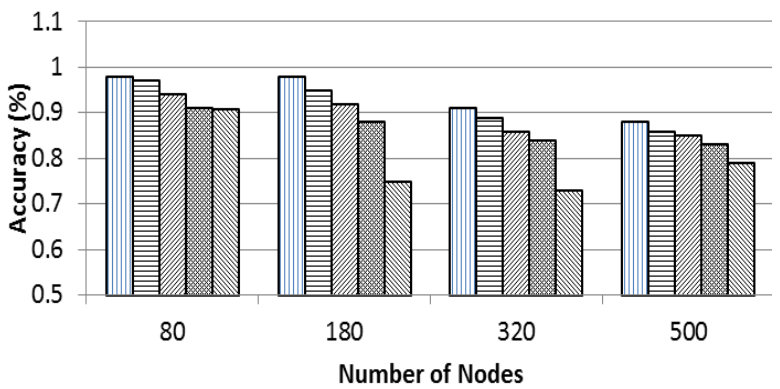

(c)

Figure 14. (a) Energy consumption; (b) latency; and (c) accuracy.

\subsubsection{Different Data Distribution}

Similarly, Four different data distributions named as uniform, center, lower bound and upper bound distribution (Section 4.1) were considered with the same variation of the network field sizes where the value of $\alpha$ was 9. Figure 15a presents the average energy consumption by each node in different distribution. It is evident that energy consumption in uniform data distribution grows radically. This is because, the data are distributed uniformly through every sector and hence each possible sector (in this case, an average nine neighboring sectors) needed to be scanned to find the target query. The other three distributions had data concentrated in a particular portion of the network. Hence, EDDS required no transmission for the null sector, as they did not store any data. Thus, energy consumption was relatively lower than the uniform distribution. However, center and lower bound distribution have almost similar energy consumption while upper bound distribution falls in the middle. The distribution presented in Figure 12 gives the rational of this behavior. Figure 15b shows the latency performance of EDDS in different distribution. Based on the aforementioned reasoning, it is obvious that the latency in uniform distribution is higher compared to other distribution. Average latency of other three distributions is almost same. Figure 15c shows the accuracy of EDDS in different distributions. It is observed that, accuracy of EDDS drops sharply with the increase of the size of the network. The accuracy of EDDS varies in between $70 \%$ and $96 \%$ at uniform distribution, $85 \%-99 \%$ at center and lower bound distribution and $80 \%-99 \%$ at upper bound distribution. 


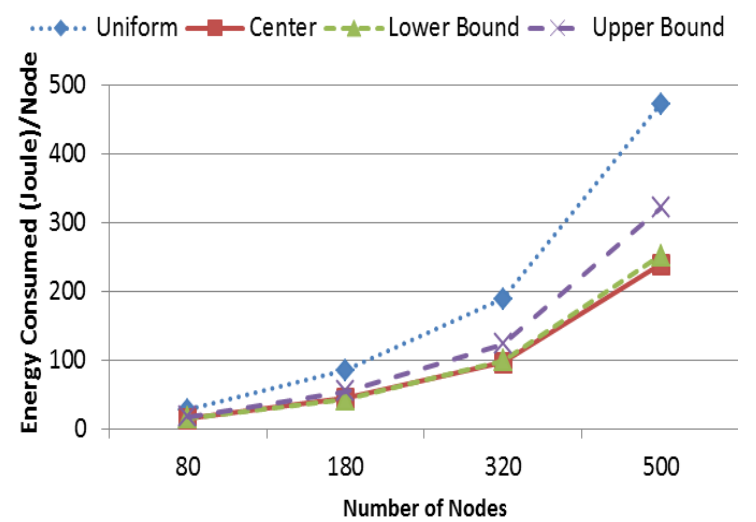

(a)

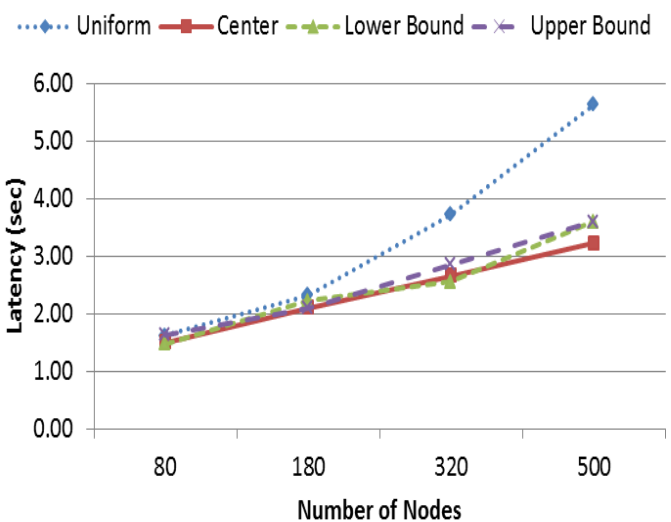

(b)

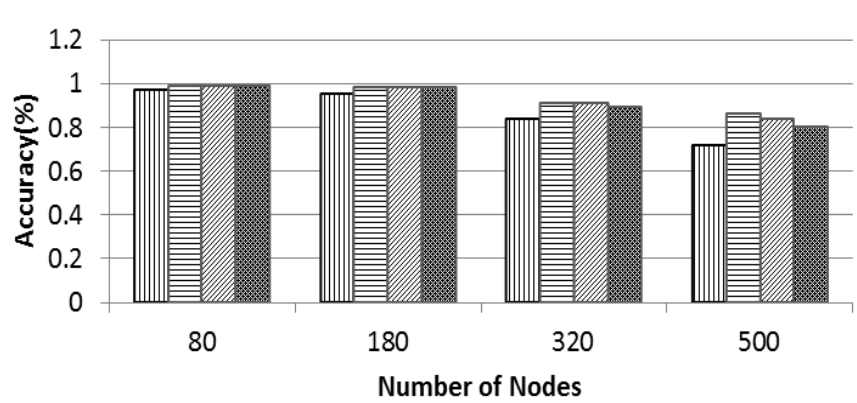

(c)

Figure 15. (a) Energy consumption; (b) latency; and (c) accuracy.

\subsection{Evaluation against SkySensor}

In this section, EDDS is evaluated against SkySensor in terms of data loss, data uniformity, success rate of the skyline query and resilience to node failure. All subsequent experiments were conducted with a network size of $150 \times 150 \mathrm{~m}^{2}$ with 500 nodes deployed uniformly. The dimension of attribute varied from 2 to 7 , data and query generation rate varied from 0.02 packets/node/second to 0.1 packets/node/second. The default value of data and query rate was 0.02 packets/node/second unless otherwise stated.

\subsubsection{Data Uniformity}

In this experiment, data uniformity of SkySensor and EDDS is studied by varying the dimension of attribute from 2 to 7 . Uniformity metric is defined as the ratio between the number of nodes used for data storage and total number of nodes in the network. In SkySensor, the number of clusters in a sensor network depends on the number of attributes of a tuple regardless of the size of the network. This is one of the major limitations of SkySensor. On the other hand, referring to Table 4, the number of sectors in EDDS for this particular network is 100, which means data are stored in 100 equivalent clusters of SkySensor resulting uniform distribution of data throughout the network. Figure 16a shows that data uniformity of SkySensor is stringently dependent on the number of dimension while this is independent and consistent for EDDS. 


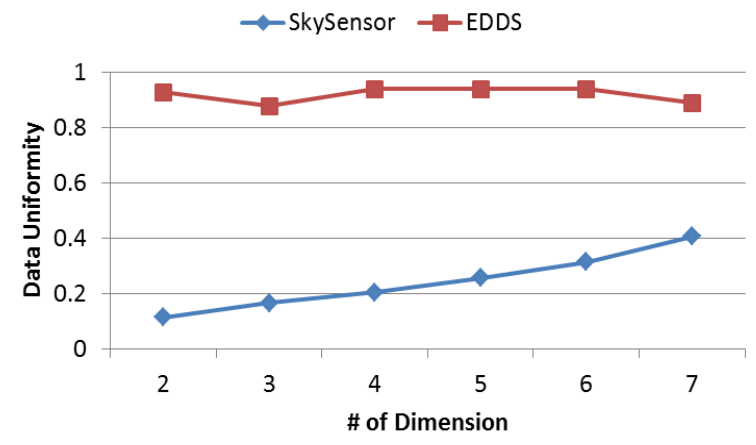

(a)

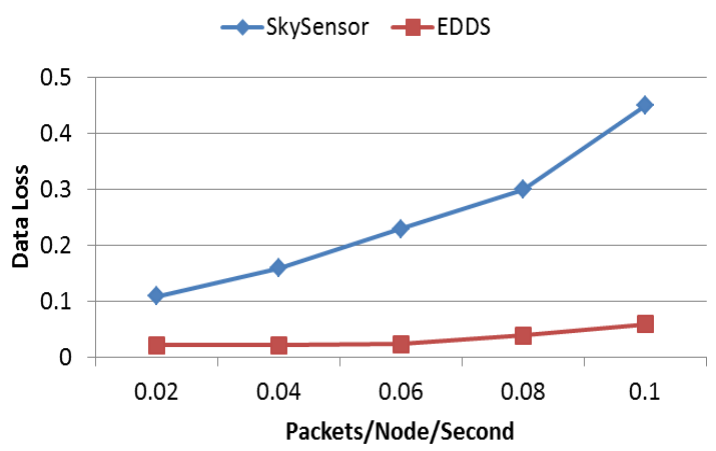

(b)

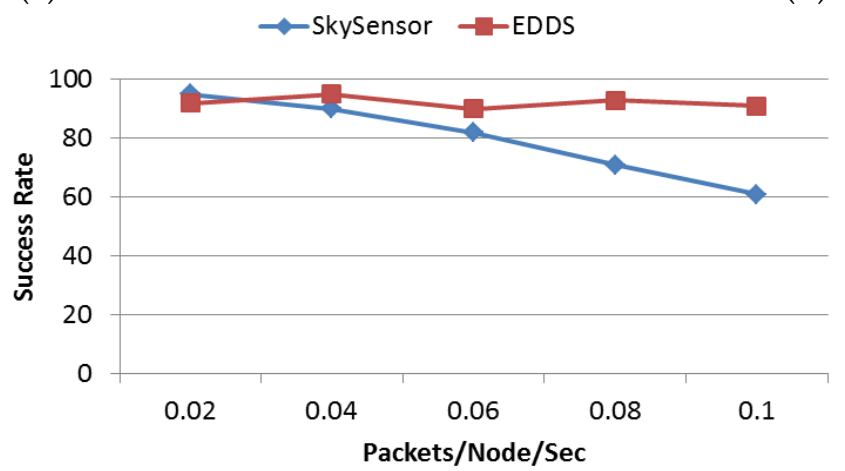

(c)

Figure 16. (a) Data uniformity; (b) data loss; and (c) success rate.

\subsubsection{Data Loss}

In this experiment, dimension of attribute was fixed at 2 and data generation rate was varied from 0.02 packets/node/second to 0.1 packets/node/second. Data loss metric is defined as the ratio of the number of data packets actually stored to the total number of packets generated. From Figure 16b, it can be seen that the percentage of data loss in SkySensor increases with an increasing data rate. This happens because there are only two clusters in SkySensor, which is clearly insufficient if the application or simulation runs for a long time. In addition, with a higher data generation rate, a hot spot or bottleneck would be created surrounding the cluster or edge nodes. On the other hand, regardless of the number of dimensions, the number of sectors or clusters is fixed ensuring an increased participation of nodes in data storage with uniform distribution.

\subsubsection{Success Rate}

Success rate in retrieving a true skyline result is studied by varying the query rate. In this experiment, the number of dimensions in attribute space was fixed at 3 . The query rate was varied from 0.02 packets/node/second to 0.1 packets/node/second. Queries were generated uniformly from different parts of the network. From Figure 16c, it is interesting to note that with a low query rate, the success rate of SkySensor is slightly higher than EDDS. However, this result reversed when the query rate was over 0.04 packets/node/second and then the success rate of SkySensor reduced as the query rate increased. This occurred due to the high concentration of all sensor readings around three clusters that eventually created congestion. A significant number of query requests and query responses were lost due to the interference, congestion and hotspot around the edge or gateway of the cluster.

\subsubsection{Resilience to Node Failure}

In this experiment, the resilience to node failure of EDDS is studied against SkySensor. In SkySensor a local replication method is adopted in order to circumvent data loss due to a storage node failure. On the other hand, EDDS integrates a Decentralized Distributed Erasure Coding (DDEC) 
algorithm instead of simple replication to achieve similar level of reliability with less redundancy [33]. In this study, the number of dimensions in attribute space was fixed at five and a fraction $f$ of nodes from the network were removed at random point of simulation. The simulation was run for 20 rounds.

In the first sub-experiment (Figure 17a), the data generation rate was varied from 0.02 to 0.1 packets/node/second and $f$ was fixed at $20 \%$. Figure 17a shows that DDEC consumed almost $50 \%$ less resources to maintain a similar or even higher magnitude of redundancy in a normal situation (packet rate $<0.08$ ). However, due to the limited number of clusters, the storage space of SkySensor depleted with packet rate greater than 0.08 packets/node/second.

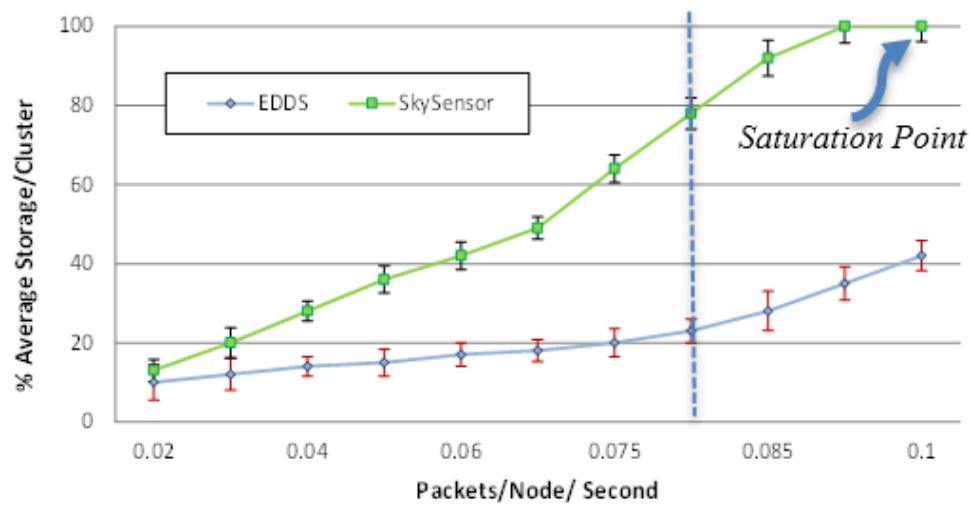

(a)

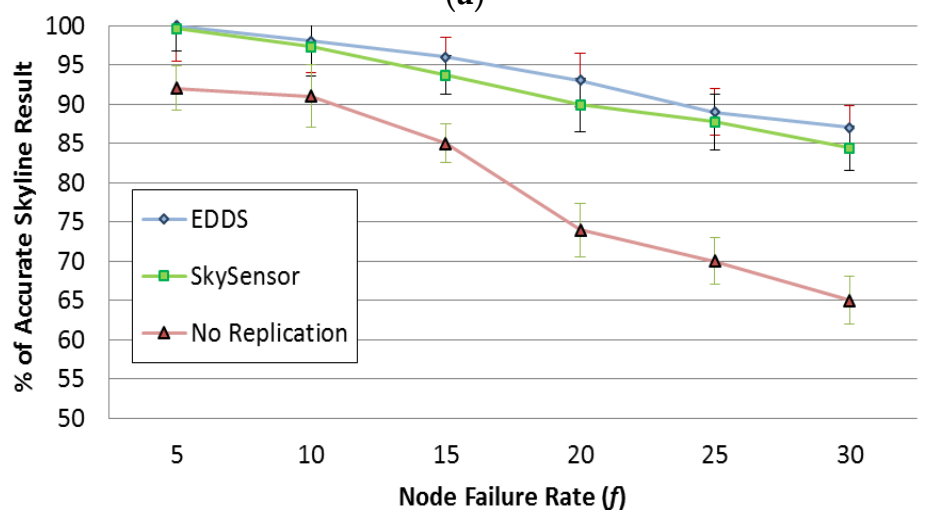

(b)

Figure 17. (a) Storage efficiency; (b) Resilience to node failure

In the second sub-experiment, data generation rate was fixed at 0.05 packets/node/second and $f$ was varied from $5 \%$ to $30 \%$. Figure $17 \mathrm{~b}$ shows the percentage of the correct skyline result after a query is issued to the network at the end of each round. It is shown that it is possible to get false skyline results. It is shown in Figure 17b that EDDS performance is similar to SkySensor under a lower data production rate. Therefore, EDDS achieves similar or higher level of reliability with less redundancy.

\section{Conclusions and Future Work}

This paper proposes a distributed approach for resolving DS efficiently in DCS of WSN. To our knowledge, this is the first practical demonstration of DS in DCS of current state-of-the-art WSN. The key feature of EDDS is the dynamic flexibility provided when carrying out queries from distributed data sources. The approach highlights the potential for using distributed skyline queries in the case of DCS of WSN and also shows the relationship between the query efficiency and storage pattern. The experiments provide results for varying WSN configurations and provide detail on the effectiveness of the dynamic skyline query approach. The current research focus is on improving EDDS for large networks and future work includes consideration of reliability and resiliency. 
Acknowledgments: This work was supported by Victorian International Research Scholarship (VIRS) and RMIT School of Electrical and Computer Engineering (SECE).

Author Contributions: This paper is one of the major outcomes of Khandakar Ahmed's PhD thesis. Khandakar contributed to the original ideas, developing the prototype in Castalia, data analysis and manuscript drafting. Nazmus Shaker Nafi contributed to manuscript drafting and revision of manuscript. Mark Gregory contributed to original ideas and revision of manuscript.

Conflicts of Interest: The authors declare no conflict of interest.

\section{References}

1. Madden, S.; Franklin, M.J.; Hellerstein, J.M.; Hong, W. Tag: A Tiny Aggregation Service for Ad-Hoc Sensor Networks. SIGOPS Oper. Syst. Rev. 2002, 36, 131-146. [CrossRef]

2. Silberstein, A.S.; Braynard, R.; Ellis, C.; Munagala, K.; Jun, Y. A sampling-based approach to optimizing top-k queries in sensor networks. In Proceedings of the 22nd International Conference on Data Engineering, Atlanta, Georgia, USA, 3-7 April 2006.

3. Wu, M.; Xu, J.; Tang, X.; Lee, W.-C. Top-k monitoring in wireless sensor networks. IEEE Trans. Knowl. Data Eng. 2007, 19, 962-976. [CrossRef]

4. Roh, Y.J.; Inchul, S.; Joo Hyuk, J.; Kyoung Gu, W.; Myoung Ho, K. Energy-efficient two-dimensional skyline query processing in wireless sensor networks. In Proceedings of the Consumer Communications and Networking Conference (CCNC), Las Vegas, NV, USA, 11-14 January 2013; pp. 294-301.

5. Borzsony, S.; Kossmann, D.; Stocker, K. The skyline operator. In Proceedings of the 17th International Conference on Data Engineering, Washington, DC, USA, 2-6 April 2001; pp. 421-430.

6. Dellis, E.; Seeger, B. Efficient computation of reverse skyline queries. In Proceedings of the 33rd International Conference on Very Large Data Bases, Vienna, Austria, 23-28 September 2007; pp. 291-302.

7. Deng, K.; Zhou, X.; Shen, H.T. Multi-source skyline query processing in road networks. In Proceedings of the IEEE 23rd International Conference on Data Engineering, Istanbul, Turkey, 11-15 April 2007; pp. 796-805.

8. Papadias, D.; Tao, Y.; Fu, G.; Seeger, B. An optimal and progressive algorithm for skyline queries. In Proceedings of the 2003 ACM SIGMOD International Conference on Management of data, San Diego, CA, USA, 9-12 June 2003; pp. 467-478.

9. Chen, B.; Liang, W.; Yu, J. Energy-efficient skyline query optimization in wireless sensor networks. Wirel. Netw. 2012, 18, 985-1004. [CrossRef]

10. Wang, G.; Xin, J.; Chen, L.; Liu, Y. Energy-efficient reverse skyline query processing over wireless sensor networks. IEEE Trans. Knowl. Data Eng. 2012, 24, 1259-1275. [CrossRef]

11. Pripužić, K.; Belani, H.; Vuković, M. Early forest fire detection with sensor networks: Sliding Window Skylines Approach. In Knowledge-Based Intelligent Information and Engineering Systems; Lovrek, I., Howlett, R., Jain, L., Eds.; Springer: Berlin, Germany, 2008; Volume 5177, pp. 725-732.

12. Shen, H.; Zhao, L.; Li, Z. A distributed spatial-temporal similarity data storage scheme in wireless sensor networks. IEEE Trans. Mob. Comput. 2011, 10, 982-996. [CrossRef]

13. Albano, M.; Chessa, S.; Nidito, F.; Pelagatti, S. Dealing with nonuniformity in data centric storage for wireless sensor networks. IEEE Trans. Parallel Distrib. Syst. 2011, 22, 1398-1406. [CrossRef]

14. Ahmed, K.; Gregory, M.A. Techniques and challenges of data centric storage scheme in wireless sensor network. J. Sens. Actuator Netw. 2012, 1, 59-85. [CrossRef]

15. Ahmed, K.; Gregory, M.A. Optimized tdma based distance routing for data centric storage. In Proceedings of the IEEE 3rd International Conference on Networked Embedded Systems for Every Application (NESEA), Liverpool, UK, 13-14 December 2012; pp. 1-7.

16. Ahmed, K.; Gregory, M.A. Wireless sensor network data centric storage routing using castalia. In Proceedings of the Telecommunication Networks and Applications Conference (ATNAC), Brisbane, Australia, 7-9 November 2012; pp. 1-8.

17. Jagadish, H.V.; Ooi, B.C.; Tan, K.-L.; Yu, C.; Zhang, R. Idistance: An Adaptive b+-Tree Based Indexing Method for Nearest Neighbor Search. ACM Trans. Database Syst. 2005, 30, 364-397. [CrossRef]

18. Chomicki, J.; Godfrey, P.; Gryz, J.; Liang, D. Skyline with presorting: Theory and Optimizations. In Intelligent Information Processing and Web Mining; Kłopotek, M., Wierzchon, S., Trojanowski, K., Eds.; Springer: Berlin, Germany, 2005; Volume 31, pp. 595-604. 
19. Godfrey, P.; Shipley, R.; Gryz, J. Maximal vector computation in large data sets. In Proceedings of the 31st International Conference on Very Large Data Bases, Trento, Italy, 4-6 October 2005; pp. 229-240.

20. Tan, K.-L.; Eng, P.-K.; Ooi, B.C. Efficient Progressive Skyline Computation; VLDB: Rome, Italy, 2001; pp. 301-310.

21. Kossmann, D.; Ramsak, F.; Rost, S. Shooting stars in the sky: An online algorithm for skyline queries. In Proceedings of the 28th International Conference on Very Large Data Bases, Hong Kong, China, 20-23 August 2002; pp. 275-286.

22. Papadias, D.; Tao, Y.; Fu, G.; Seeger, B. Progressive skyline computation in database systems. ACM Trans. Database Syst. 2005, 30, 41-82. [CrossRef]

23. Liang, W.; Chen, B.; Yu, J.X. Energy-efficient skyline query processing and maintenance in sensor networks. In Proceedings of the 17th ACM Conference on Information and Knowledge Management, Napa Valley, CA, USA, 26-30 October 2008; pp. 1471-1472.

24. Xin, J.; Wang, G.; Chen, L.; Zhang, X.; Wang, Z. Continuously maintaining sliding window skylines in a sensor network. In Advances in Databases: Concepts, Systems and Applications; Kotagiri, R., Krishna, P.R., Mohania, M., Nantajeewarawat, E., Eds.; Springer: Berlin, Germany, 2007; Volume 4443, pp. 509-521.

25. Baichen, C.; Weifa, L. Progressive skyline query processing in wireless sensor networks. In Proceedings of the 5th International Conference on Mobile Ad-hoc and Sensor Networks, MSN '09, Fujian, China, 14-16 December 2009; pp. 17-24.

26. Song, S.; Kwak, Y.; Lee, S. Skyline query processing in sensor network based on data centric storage. Sensors 2011, 11, 10283-10292. [CrossRef] [PubMed]

27. Aly, M.; Pruhs, K.; Chrysanthis, P.K. Kddcs: A Load-Balanced in-Network Data-Centric Storage Scheme for Sensor Networks. In Proceedings of the 15th ACM International Conference on Information and Knowledge Management, Arlington, VA, USA, 5-11 November 2006; pp. 317-326.

28. Song, S.; Bok, K.; Kwak, Y.S.; Goo, B.; Kwak, Y.; Ko, D. Dynamic load balancing data centric storage for wireless sensor networks. Sensors 2010, 10, 10328-10338. [CrossRef] [PubMed]

29. Li, X.; Kim, Y.J.; Govindan, R.; Hong, W. Multi-dimensional range queries in sensor networks. In Proceedings of the 1st International Conference on Embedded Networked Sensor Systems, Los Angeles, CA, USA, 5-7 November 2003; pp. 63-75.

30. Su, I.F.; Chung, Y.-C.; Lee, C.; Lin, Y.-Y. Efficient skyline query processing in wireless sensor networks. J. Parallel Distrib. Comput. 2010, 70, 680-698. [CrossRef]

31. OMNeT++Community. Omnet++. Available online: http://www.omnetpp.org/ (accessed on 5 May 2014).

32. Wei, Y.; Heidemann, J.; Estrin, D. An energy-efficient mac protocol for wireless sensor networks. In Proceedings of the Twenty-First Annual Joint Conference of the IEEE Computer and Communications Societies, New York, NY, USA, 23-27 June 2002; Volume 1563, pp. 1567-1576.

33. Ahmed, K.; Gregory, M.A. Decentralized coding algorithm in data centric storage for wireless sensor networks. In Proceedings of the Telecommunication Networks and Applications Conference (ATNAC), Christchurch, New Zealand, 20-22 November 2013; pp. 106-111.

(C) 2016 by the authors; licensee MDPI, Basel, Switzerland. This article is an open access article distributed under the terms and conditions of the Creative Commons by Attribution (CC-BY) license (http://creativecommons.org/licenses/by/4.0/). 